\title{
Stability and accuracy of various difference schemes for the lattice Boltzmann method
}

V. Zecevic ${ }^{1} \quad$ M. P. Kirkpatrick ${ }^{2} \quad$ S. W. Armfield ${ }^{3}$

(Received 5 January 2012; revised 30 July 2012)

\begin{abstract}
We test a second order central difference scheme and a first order upwind scheme for the advection of particles in the lattice Boltzmann method for fluid flow. A diffusion term is added to the Boltzmann equation in order to improve stability when using the second order scheme, this term is equivalent to the Lax-Wendroff scheme for a particular value of the diffusion constant. In contrast to the normal lattice Boltzmann method, we allow a particle Courant number less than one. We test the schemes for stability and accuracy using Taylor-Green vortex and channel flows in three dimensions, finding improved stability for some configurations and no loss in accuracy. Both modifications are expected to remove some spurious lattice invariants. The proposed particle diffusion term may also be used to improve the stability of other Boltzmann based methods that use higher order difference schemes.
\end{abstract}

http://journal.austms.org.au/ojs/index.php/ANZIAMJ/article/view/5073 gives this article, (c) Austral. Mathematical Soc. 2012. Published August 9, 2012. ISSN 1446-8735. (Print two pages per sheet of paper.) Copies of this article must not be made otherwise available on the internet; instead link directly to this URL for this article. 


\section{Contents}

1 Introduction

C495

2 Discretizing the Boltzmann equation $\quad$ C496

2.1 First order upwind scheme . . . . . . . . . . . . C C497

2.2 Second order central scheme . . . . . . . . . . . C498

3 Accuracy

C499

3.1 Channel flow . . . . . . . . . . . . . . . . . . . C499

3.2 Taylor-Green vortex . . . . . . . . . . . . . . C500

4 Stability

C503

4.1 Taylor-Green vortex . . . . . . . . . . . . . C504

4.2 Channel flow . . . . . . . . . . . . . . C505

4.3 Fifteen speed lattice . . . . . . . . . . . . C506

5 Discussion

C506

References

C508

\section{Introduction}

The lattice Boltzmann (LB) method is an increasingly popular means of simulating fluid flow, the explicit and local nature of calculations make it an attractive method for parallel computing. Others possible discretizations of the discrete velocity Boltzmann equation include finite volume [1, 2], finite difference [3] or finite element [4] schemes, in general proposed in order to remove the requirement of a uniform grid. It is also possible to use an interpolation supplemented scheme [5] in order to allow a non-uniform grid. Our modified schemes are more closely related to normal LB methods, the grid spacing is still uniform and neighbour nodes are still in the same direction as 
particle velocity vectors.

We test two modified difference schemes on a uniform grid in order to investigate the effects of the discretization on stability and accuracy. The first modification is to use a non-unit particle Courant number and the second is to use a second order central difference scheme for spatial derivatives. We propose adding a particle diffusion term to improve stability when using the second order central scheme. The particle diffusion increases the viscosity in a predictable way and does not degrade the accuracy of the scheme, this is equivalent to the Lax-Wendroff scheme for a particular value of the diffusion constant. Both modifications are expected to remove the staggered invariant described by Zanetti [6].

\section{Discretizing the Boltzmann equation}

The lattice Boltzmann method is one of many possible discretizations of the discrete velocity Boltzmann equation,

$$
\frac{\partial f_{\mathfrak{i}}\left(x_{\alpha}, t\right)}{\partial t}+c_{i \alpha} \partial_{\alpha} f_{\mathfrak{i}}\left(x_{\alpha}, t\right)=\Omega_{\mathfrak{i}}\left(x_{\alpha}, t\right) .
$$

The $\mathfrak{i}$ subscripts denote discrete particle populations with a probability density $f_{\mathfrak{i}}$ and velocity $c_{\mathfrak{i} \alpha}$. They are bold to distinguish them from Greek subscripts, representing spatial dimensions using summation notation. The left hand side represents the advection of particles and the collision operator $\Omega$ represents the effects of collisions between particles.

With normal LB methods, the particle velocities and grid spacing are such that in one time step, particle populations travel exactly to the next site in their path. This simplifies the implementation, allows the use of an upwind collision operator and makes it easy to quantify and correct second order diffusive discretization errors. 


\subsection{First order upwind scheme}

We take a forward Euler temporal discretization, a first order upwind spatial discretization and an upwind collision operator to obtain,

$$
\frac{f_{i}^{x, t+}-f_{i}^{x, t}}{\Delta t}+\left\|c_{i \alpha}\right\| \frac{f_{i}^{x, t}-f_{i}^{x-, t}}{\left\|\Delta x_{i \alpha}\right\|}=\Omega_{i}^{x-, t}
$$

The discrete space unit $\Delta x_{\mathfrak{i} \alpha}$ is a different vector for each particle population so is given an $\mathfrak{i}$ subscript and we use the convention

$$
f_{\mathfrak{i}}^{x \pm, t \pm}=f_{\mathfrak{i}}\left(x_{\alpha} \pm \Delta x_{\mathfrak{i} \alpha}, t \pm \Delta t\right) .
$$

Substituting in the particle Courant number,

$$
\mathrm{Cr}=\frac{\left\|\mathrm{c}_{\mathrm{i} \alpha}\right\| \Delta \mathrm{t}}{\left\|\Delta \mathrm{x}_{\mathbf{i} \alpha}\right\|}
$$

simplifies the time advancement:

$$
f_{i}^{x, t+}=(1-\mathrm{Cr}) f_{i}^{x, t}+\operatorname{Cr} f_{i}^{x-, t}+\Delta t \Omega_{i}^{x-, t} .
$$

When the Courant number is 1 , the first term on the right hand side disappears and the scheme reduces to the lattice Boltzmann method.

The commonly used single relaxation time collision operator adjusts particle populations towards their equilibrium values $f^{e q}$,

$$
\Omega_{\mathfrak{i}}\left(x_{\alpha}, t\right)=\omega\left(f_{\mathfrak{i}}^{e q}\left(x_{\alpha}, t\right)-f_{\mathfrak{i}}\left(x_{\alpha}, t\right)\right),
$$

and leads to the familiar form

$$
f_{i}\left(x_{\alpha}, t\right)=(1-\Delta t \omega) f_{i}\left(x_{\alpha}-\Delta x_{i \alpha}, \Delta t\right)+\Delta t \omega f_{i}^{e q}\left(x_{\alpha}-\Delta x_{i \alpha}, \Delta t\right) .
$$

With a suitable choice of lattice, and using a multi-scale expansion, Frisch et al. [7] shown that this method approaches the incompressible NavierStokes (NS) equations:

$$
\rho\left(\partial_{t} u_{\alpha}+u_{\beta} \partial_{\beta} u_{\alpha}\right)=-\partial_{\alpha} P+v \rho \partial_{\beta} \partial_{\beta} u_{\alpha} .
$$


The pressure is approximated by density and the viscosity is related to the relaxation factor $\omega$ and the speed of sound $c_{s}$ :

$$
\mathrm{P}=\mathrm{c}_{\mathrm{s}}^{2} \rho \quad \text { and } \quad v=\mathrm{c}_{s}^{2}\left[\frac{1}{\omega}-\frac{1}{2} \frac{\Delta \mathrm{t}}{\mathrm{Cr}}\right] .
$$

It is customary for $\Delta t, \Delta x_{i \alpha}$ and $c_{i \alpha}$ all to be set to 1 . Due to space constraints, the multi-scale expansion for our modified schemes is not included in this article.

\subsection{Second order central scheme}

We propose adding a diffusion term along particle directions to the Boltzmann equation to improve stability when using the second order central scheme:

$$
\frac{\partial f_{\mathfrak{i}}\left(x_{\alpha}, t\right)}{\partial t}+c_{i \alpha} \partial_{\alpha} f_{i}\left(x_{\alpha}, t\right)-\alpha c_{i \alpha} c_{i \beta} \partial_{\alpha} \partial_{\beta} f_{\mathfrak{i}}\left(x_{\alpha}, t\right)=\Omega_{\mathfrak{i}}\left(x_{\alpha}, t\right) .
$$

Using a forward Euler temporal discretization, second order central spatial discretizations and a downwind collision operator we obtain,

$$
\frac{f_{i}^{x, t+}-f_{i}^{x, t}}{\Delta t}+\left\|c_{i \alpha}\right\| \frac{f_{i}^{x+, t}-f_{i}^{x-, t}}{2\left\|\Delta x_{i \alpha}\right\|}-\alpha\left\|c_{i \alpha}\right\|^{2} \frac{f_{i}^{x+, t}+f_{i}^{x-, t}-2 f_{i}^{x, t}}{\left\|\Delta x_{i \alpha}\right\|^{2}}=\Omega_{i}^{x-, t} .
$$

Again we substitute the Courant number and the time advancement becomes

$$
\begin{aligned}
f_{i}^{x, t+}=f_{i}^{x, t} & \left(1-\frac{2 \alpha \mathrm{Cr}^{2}}{\Delta t}\right) \\
+ & f_{i}^{x-, t}\left(\frac{\alpha \mathrm{Cr}^{2}}{\Delta t}+\frac{\mathrm{Cr}}{2}\right)+f_{i}^{x+, t}\left(\frac{\alpha \mathrm{Cr}^{2}}{\Delta \mathrm{t}}-\frac{\mathrm{Cr}}{2}\right)+\Delta \mathrm{t} \Omega_{i}^{\mathrm{x}-, \mathrm{t}} .
\end{aligned}
$$

Performing a multi-scale expansion and using a single relaxation time collision operator we arrive at the incompressible Navier-Stokes equations again. This time the viscosity is

$$
v=c_{s}^{2}\left[\frac{1}{\omega}+\alpha-\frac{\Delta t}{\mathrm{Cr}}\right] .
$$


If $\Delta t, \Delta x_{i \alpha}$ and $c_{i \alpha}$ are all set to 1 and $\alpha=0.5$, then this scheme reduces exactly to the lattice Boltzmann method. Choosing $\alpha=0.5 \Delta t$ is equivalent to the Lax-Wendroff second order difference scheme.

We define a cell Peclet number for particle advection:

$$
\mathrm{Pe}=\frac{\left\|\Delta \mathrm{x}_{\mathfrak{i} \alpha}\right\| \cdot\left\|\mathrm{c}_{\mathfrak{i} \alpha}\right\|}{\alpha\left\|\mathrm{c}_{\mathfrak{i} \alpha}\right\|^{2}}=\frac{\left\|\Delta \mathrm{x}_{\mathfrak{i} \alpha}\right\|}{\alpha\left\|\mathrm{c}_{\mathfrak{i} \alpha}\right\|} .
$$

Neglecting the collision operator $\Omega$, we might expect stability for $\mathrm{Pe} \leqslant 2$. This is in addition to any stability concerns of the lattice Boltzmann method itself. In our experiments, $\left\|\Delta \mathrm{x}_{\mathbf{i} \alpha}\right\|=\left\|\mathrm{c}_{\mathfrak{i} \alpha}\right\|$ so the advection scheme should be stable for $\alpha \geqslant 0.5$.

\section{Accuracy}

We tested the accuracy of the normal and modified schemes by simulating a Taylor-Green vortex and channel flow and comparing the results to analytical solutions. We use the single relaxation time collision operator and the D3Q19 lattice for all simulations. Keeping in mind that the time step is not usually varied in the lattice Boltzmann method, we change the non-dimensional time step by varying the Mach number:

$$
\Delta \mathrm{t}^{*}=\Delta \mathrm{t} \frac{\mathrm{u}_{0}}{l_{0}}=\Delta \mathrm{t} \frac{\mathrm{Ma}}{\mathrm{l}_{0}} \mathrm{c}_{\mathrm{s}} .
$$

\subsection{Channel flow}

The first test was for steady state error of a laminar channel flow simulated in three spatial dimensions. The flow was initialised to the analytical solution and left to evolve until it reached a steady state. Trials were performed at a range of Mach numbers and grid sizes shown in Table 1 with the Reynolds 
Table 1: Parameters tested for laminar channel flow.

\begin{tabular}{lrrrrr} 
Channel height & 64 & 96 & 128 & 160 & 192 \\
Mach number & 0.10 & 0.08 & 0.06 & 0.04 & 0.02 \\
\hline
\end{tabular}

number fixed at one. Each simulation was repeated using three discretizations. First the normal lattice Boltzmann method, labelled as 'upwind, $d t=1$ ', then the first order difference scheme with a Courant number of 0.5 labelled as 'upwind, $\mathrm{dt}=0.5$ ' and finally the second order scheme with $\alpha=0.5 \Delta \mathrm{t}$ and $\mathrm{Cr}=0.5$ labelled as 'Lax-Wendroff, $\mathrm{dt}=0.5$ '.

The error behaviour is linear with respect to Mach number (Figure 1) and we compare the predicted error as Mach number (time step) approaches zero for each grid size in Figure 2 finding second order slope for all schemes. Both first order schemes have exactly the same error magnitude while the second order scheme had approximately half the error.

\subsection{Taylor-Green vortex}

The Taylor-Green (TG) vortex is an unsteady flow with gradients in all directions in contrast to channel flow which is steady in time and only has a velocity gradient in one direction. We further tested the Mach number error dependence using this flow by comparing the $\mathrm{L}^{2}$ norm of the error to the analytical solution

$$
\begin{aligned}
& u(x, y, t)=u_{0} e^{-2 k_{0}^{2} v t} \cos \left(k_{0} x\right) \sin \left(k_{0} y\right), \\
& v(x, y, t)=-u_{0} e^{-2 k_{0}^{2} v t} \sin \left(k_{0} x\right) \cos \left(k_{0} y\right), \\
& P(x, y, t)=P_{0}+\frac{u_{0}^{2}}{4} e^{-4 k_{0}^{2} v t} \cos \left(2 k_{0} x\right) \cos \left(2 k_{0} y\right) .
\end{aligned}
$$

The flow has a characteristic time $t^{*}$ and wavenumber $k_{0}$ :

$$
\mathrm{t}^{*}=2 \mathrm{k}_{0}^{2} v \mathrm{t} \text { and } \mathrm{k}_{0}=\mathrm{l}_{0}^{-1} .
$$



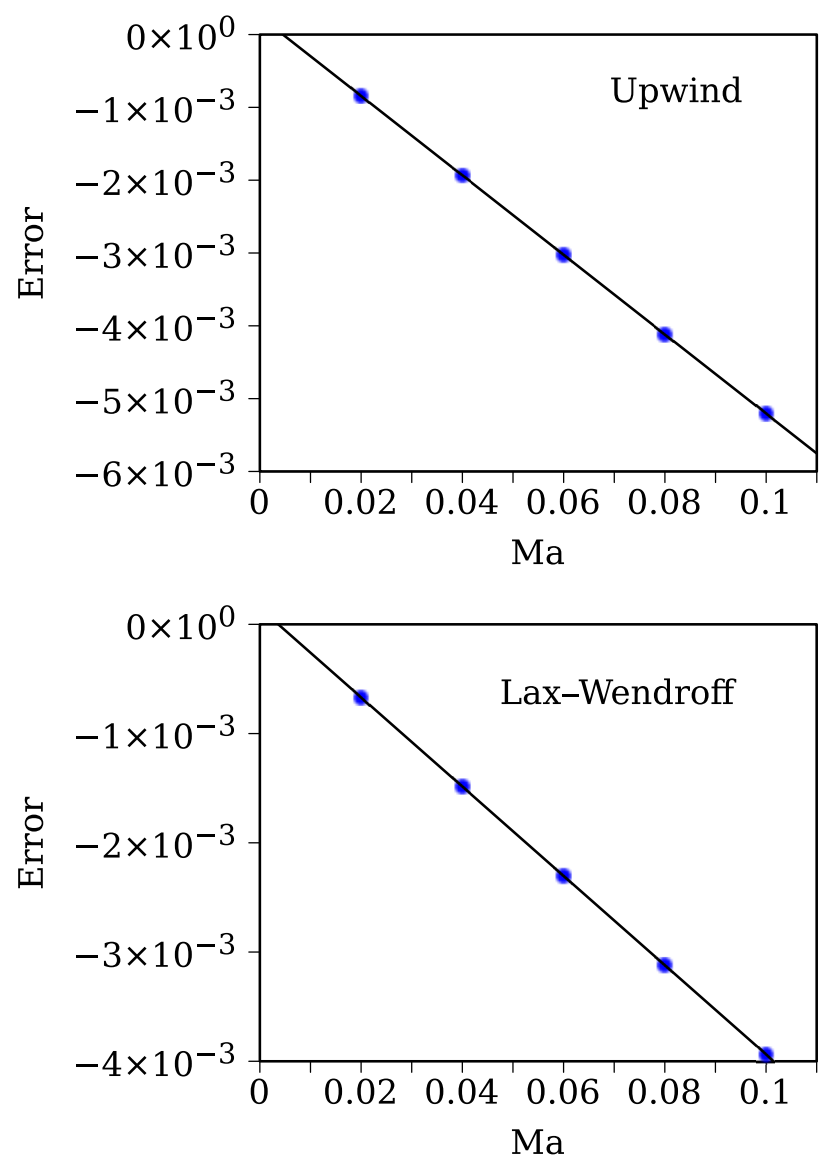

Figure 1: Mach number dependence with the normal scheme (above) and the second order Lax-Wendroff scheme (below) for laminar channel flow. 


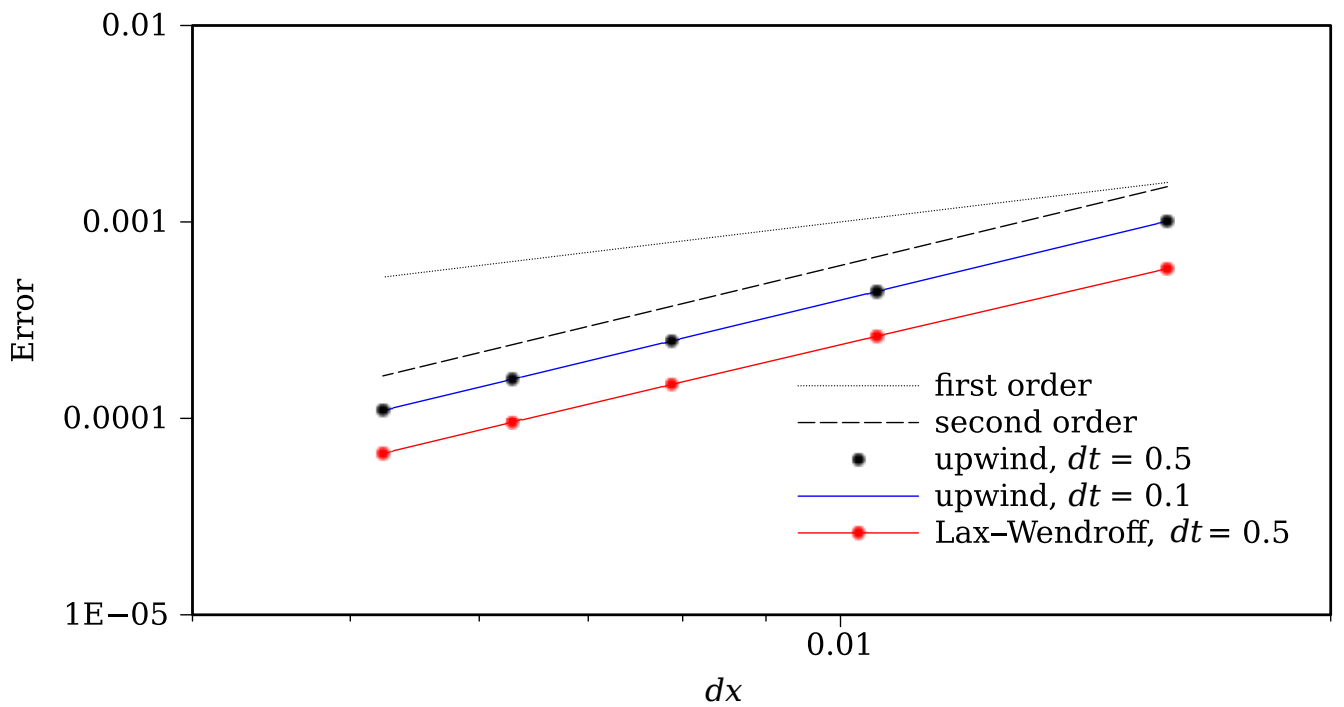

Figure 2: Spatial accuracy using 3D laminar channel flow, zero Mach number extrapolation.

The same three schemes from the channel flow simulation were used again. For all tests, the Reynolds number was kept at one and the domain was a 128 node cube. The error was measured at a non dimensional time, $t^{*}=1.3863$, chosen so that the characteristic velocity has reduced by a factor of four by this time.

The results, shown in Figure 3, show an order of accuracy that varies between first and second order depending on Mach number. The results are slightly inconsistent with channel flow simulations which showed a purely first order Mach number dependence. The upwind scheme with reduced Courant number had the lowest error at small Mach numbers while the Lax-Wendroff scheme had slightly worse error. The Lax-Wendroff scheme was unable to complete the lowest Mach number simulation due to instability. 


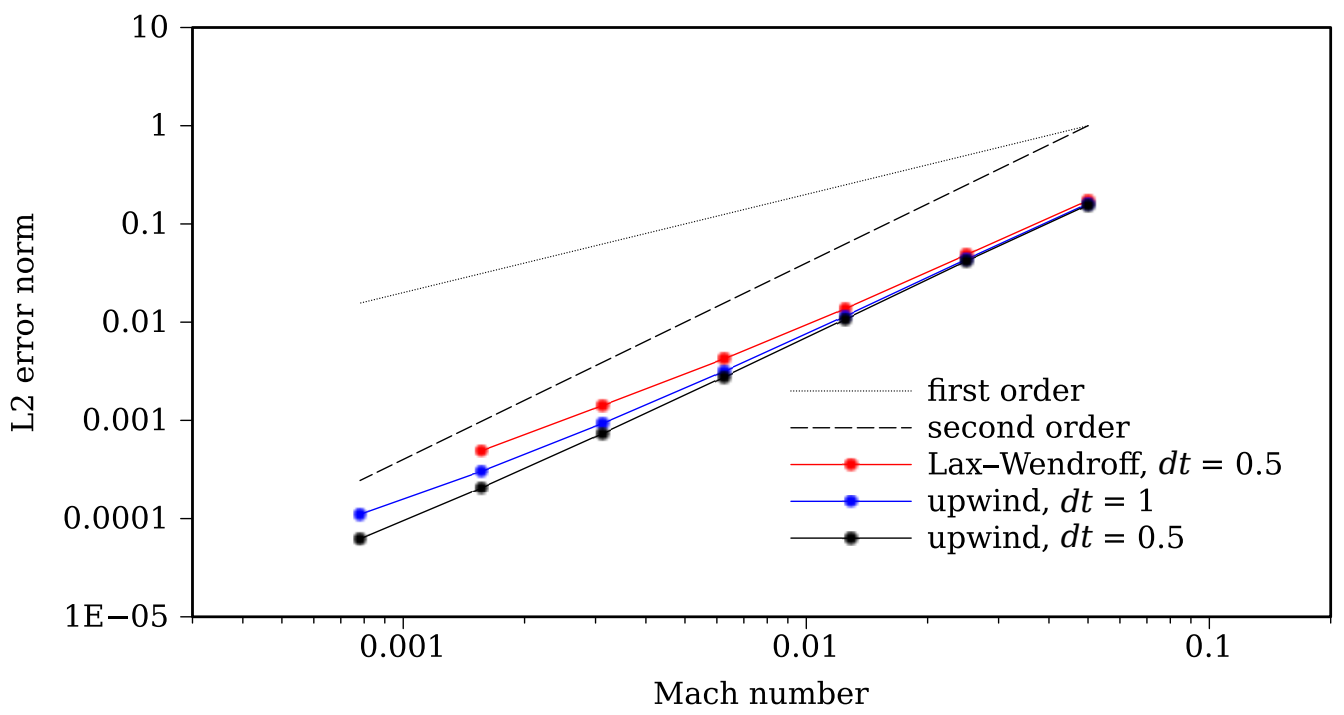

Figure 3: Taylor-Green vortex simulation, $\mathrm{L}^{2}$ norm of error at non-dimensional time $\mathrm{t}^{*}=1.3863$.

\section{Stability}

The stability of the modified and original schemes was tested by performing the same simulations at an increased Reynolds number. We have previously verified that the normal lattice Boltzmann method successfully approaches the correct statistics for turbulent channel flow [8] reaching similar conclusions to Bespalko, Pollard and Uddin [9]. Initial simulations were performed using the D3Q19 lattice and Section 4.3 covers some additional experiments using the D3Q15 lattice. 
Table 2: Stability results for TG vortex.

\begin{tabular}{ccccc}
\hline Re & Normal & $\mathrm{Cr}=0.5$ & $\begin{array}{c}\text { Central } \\
\alpha=0.491\end{array}$ & $\begin{array}{c}\text { Central } \\
\alpha=0.492\end{array}$ \\
\hline 10,000 & stable & stable & unstable & stable \\
15,000 & unstable & unstable & - & unstable \\
\hline
\end{tabular}

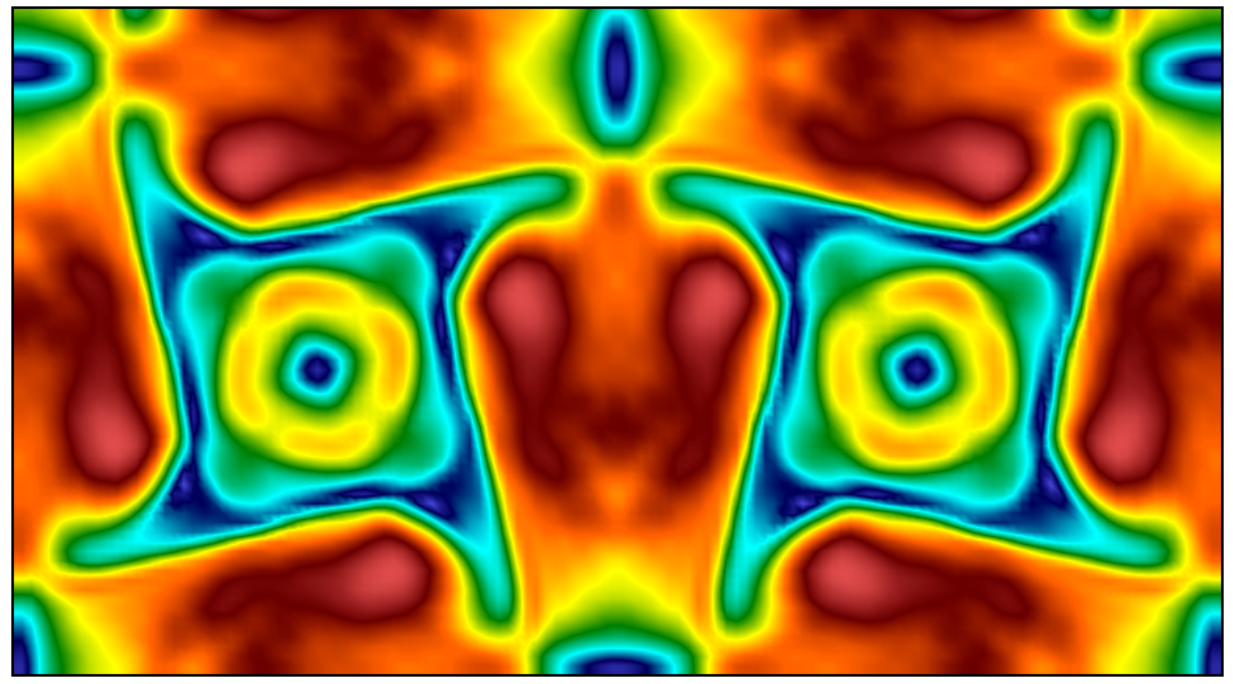

Figure 4: Velocity magnitude plot of TG vortex at $\operatorname{Re}=10,000$ and $t=$ 10,500 .

\subsection{Taylor-Green vortex}

All tests of the TG vortex were done using a 128 node cube grid and a Mach number of 0.05. Initial experiments with the second order central scheme found that $\alpha=0.492$ was the minimum required for stability with a Reynolds number of 10,000. Next, all discretizations were run at $R e=15,000$ and found to be unstable. Hence, the modified schemes do not degrade or enhance stability for the turbulent TG vortex. The results are summarised in Table 2 and an example of the flow field is shown in Figure 4. 
Table 3: Stability results for turbulent channel flow using bounce back, Guo, and equilibrium (Eq) boundary conditions. Time step when flow field becomes non-physical shown for unstable runs.

\begin{tabular}{cccccc}
\hline $\mathrm{Re}_{\tau}$ & \multicolumn{2}{c}{ Bounce back } & \multicolumn{2}{c}{$\mathrm{Guo}$} & $\mathrm{Eq}$ \\
& normal & $\mathrm{Cr}=0.8$ & normal & $\mathrm{Cr}=0.8$ & \\
\hline 180 & stable & stable & stable & stable & stable \\
380 & 18,000 & stable & 5,500 & stable & stable \\
560 & - & stable & - & 40,000 & stable \\
\hline
\end{tabular}

\subsection{Channel flow}

The channel flow was tested at a range of wall Reynolds numbers

$$
\operatorname{Re}_{\tau}=\frac{u_{\tau} \delta}{v} \quad \text { where, } \quad u_{\tau}=\sqrt{\left.v \frac{\partial u}{\partial y}\right|_{y=0}} .
$$

The stability was found to depend on boundary conditions. We tested three different implementations of the solid wall boundary. The simplest is the bounce back boundary condition. Here the solid wall bounces particles back along their incoming direction. Another method is to set the equilibrium and off-equilibrium parts of the distribution separately for boundary nodes as proposed by Guo et al. [10]. The equilibrium part is calculated from the prescribed velocity while the off-equilibrium part is copied from the nearest neighbour node. It is also possible to simply set the boundary nodes to equilibrium values at the expense of accuracy.

The stability results were the same for the first order upwind and second order central schemes when $\mathrm{Cr}=0.8$ so separate results are not shown. The highest achievable Reynolds number was increased for the Guo and bounce back boundary conditions by using the modified schemes. The equilibrium boundary condition was stable over all Reynolds numbers even with the normal discretization. This indicates that the dominant instability is related to the boundary condition used. 

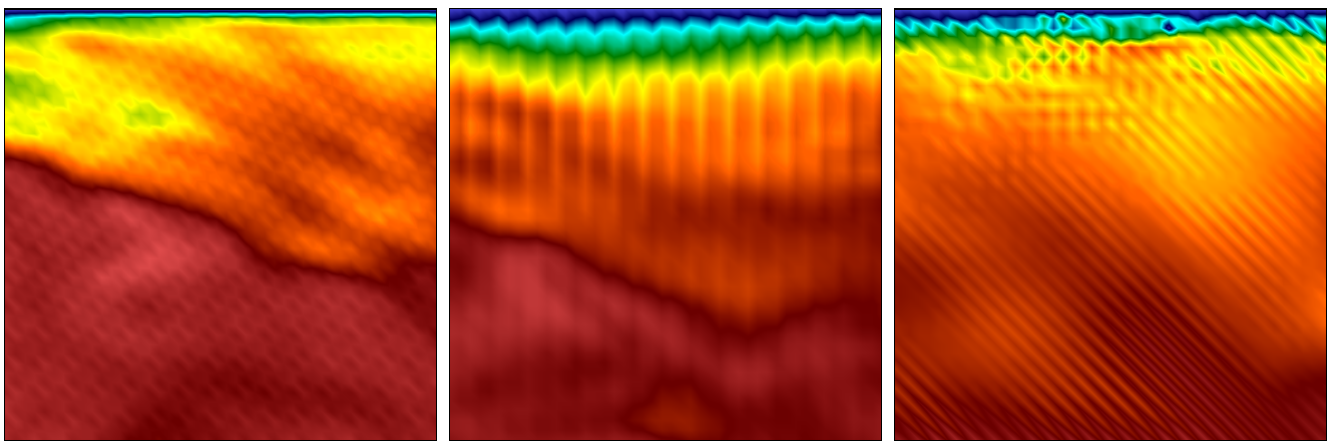

Figure 5: Velocity magnitude plot showing instabilities for bounce back BC (left), Guo BC (middle) and bounce back BC with D3Q15 lattice (right).

\subsection{Fifteen speed lattice}

Previous results were obtained using the D3Q19 lattice. We also tested the less computationally intense D3Q15 lattice. Results for the TG vortex showed no difference in stability while the channel flow showed much worse stability with the fifteen speed lattice. Simulations using this lattice quickly became unstable even at $\operatorname{Re}_{\tau}=180$ using the Guo and bounce back boundary conditions. Although the equilibrium boundary condition was stable with this lattice, it showed visible artifacts. The modified schemes with $\mathrm{Cr}=0.8$ removed all artifacts and allowed stable simulation using all boundary conditions at $\operatorname{Re}_{\tau}=180$.

\section{Discussion}

As expected the second order central scheme was generally not stable without any diffusion. Even with the correct amount of diffusion so that the scheme was equivalent to the Lax-Wendroff scheme, Taylor-Green vortex simulations with $\operatorname{Re}=1$ were not stable over the whole range of parameters. Unstable 
simulations would proceed for some time with non-physical wiggles setting in eventually. The minimum amount of diffusion required to ensure stability varied depending on the flow configuration and as predicted, was always below $\alpha=0.5$ approaching this value for turbulent flows. Thus, the proposed method of adding particle diffusion stabilised the second order central scheme to the point where it had similar stability properties to other schemes for the flows investigated.

Reducing the particle Courant number below one allows significant stability improvements for the simulation of turbulent channel flow. One explanation for the stability behaviour may be due to lattice invariants, mentioned by Zanetti [6] when dealing with lattice gas cellular automata. Ginzburg et al. [11] and d'Humieres et al. [12] gave a more recent discussion and note that the effects depend on the boundary condition used. In general, these invariants arise due to particle populations moving discretely from one lattice site to another, so using a Courant number smaller than one removes them. Fractional propagation was first suggested by Qian [13] as a means of removing lattice invariants from one dimensional cellular automata (an early predecessor to LB methods).

The two modified schemes presented in this article simulate the incompressible Navier-Stokes equations with at least the same accuracy as the normal lattice Boltzmann method. The normal first order discretization with a Courant number smaller than one may provide increased stability for any simulation using solid wall boundary conditions particularly if the D3Q15 lattice is to be used. The proposed particle diffusion term may also find application for other discretizations of the Boltzmann equation where higher order differencing is desirable.

Acknowledgements This work has been supported by the Australian Research Council and by an Australian Postgraduate Award. 


\section{References}

[1] F. Nannelli and S. Succi. The lattice Boltzmann equation on irregular lattices. J. Stat. Phys., 68(3-4):401-407, Aug 1992.

doi:10.1007/BF01341755. Nato Advanced Research Workshop : Lattice Gas Automata, Theory, Implementation, Simulations, Nice, France, Jun 25-28, 1991. C495

[2] G. W. Peng, H. W. Xi, C. Duncan, and S. H. Chou. Finite volume scheme for the lattice boltzmann method on unstructured meshes. Phys. Rev. E, 59(4):4675-4682, Apr 1999. doi:10.1103/PhysRevE.59.4675. $\mathrm{C} 495$

[3] N. Z. Cao, S. Y. Shen, S. Jin, and D. Martinez. Physical symmetry and lattice symmetry in the lattice Boltzmann method. Phys. Rev. E, 55(1, Part a):R21-R24, Jan 1997. doi:10.1103/PhysRevE.55.R21. C495

[4] T. Lee and C. L. Lin. A characteristic Galerkin method for discrete Boltzmann equation. J. Comput. Phys., 171(1):336-356, July 2001. doi:10.1006/jcph.2001.6791. C495

[5] X. Y. He and G. D. Doolen. Lattice Boltzmann method on a curvilinear coordinate system: Vortex shedding behind a circular cylinder. Phys. Rev. E, 56(1, Part a):434-440, Jul 1997. doi:10.1103/PhysRevE.56.434. C495

[6] G. L. Zanetti. Hydrodynamics of lattice-gas automata. Phys. Rev. A, 40(3):1539-1548, Aug 1989. doi:10.1103/PhysRevA.40.1539. C496, C507

[7] U. Frisch, D. d'Humieres, B. Hasslacher, P. Lallemand, Y. Pomeau, and J. P. Rivet. Lattice gas hydrodynamics in two and three dimensions. Complex Systems, 1:649-707, 1987. C497

[8] V. Zecevic, M. P. Kirkpatrick, and S. W. Armfield. The lattice Boltzmann method for turbulent channel flows using graphics processing 
units. ANZIAM J., 52(0):914-928, 2011. http://journal .austms.org. au/ojs/index.php/ANZIAMJ/article/view/3951. C503

[9] D. Bespalko, A. Pollard, and M. Uddin. Direct numerical simulation of fully-developed turbulent channel flow using the lattice Boltzmann method and analysis of OpenMP scalability. In High Performance Computing Systems and Applications, volume 5976 of Lect. Notes Comput. Sci., pages 1-19. Springer Berlin, 2010. doi:10.1007/978-3-642-12659-8_1. C503

[10] Z. L. Guo, C. G. Zheng, and B. C. Shi. An extrapolation method for boundary conditions in lattice Boltzmann method. Phys. Fluids, 14(6):2007-2010, Jun 2002. doi:10.1063/1.1471914. C505

[11] I. Ginzburg, F. Verhaeghe, and D. d'Humieres. Two-relaxation-time lattice Boltzmann scheme: About parametrization, velocity, pressure and mixed boundary conditions. Commun. Comput. Phys., 3(2):427-478, Feb 2008. C507

[12] D. d'Humieres, I. Ginzburg, M. Krafczyk, P. Lallemand, and L. S. Luo. Multiple-relaxation-time lattice boltzmann models in three dimensions. Philos. Trans. R. Soc. Lond. Ser. A-Math. Phys. Eng. Sci., 360(1792):437-451, Mar 2002. doi:10.1098/rsta.2001.0955. C507

[13] Y. H. Qian. Fractional propagation and the elimination of staggered invariants in lattice-bgk models. Int. J. Mod. Phys. C, 8(4):753-761, Aug 1997. doi:10.1142/S0129183197000643. 6th International Conference on Discrete Models for Fluid Mechanics, Boston Univ, Ctr. Computat. Sci, Boston, MA, Aug 26-28, 1996. C507

\section{Author addresses}

1. V. Zecevic, School of Aerospace, Mechanical and Mechatronic Engineering, The University of Sydney, Sydney, Australia. 
mailto:vanja.zecevic@sydney.edu.au

2. M. P. Kirkpatrick, School of Aerospace, Mechanical and Mechatronic Engineering, The University of Sydney, Sydney, Australia.

3. S. W. Armfield, School of Aerospace, Mechanical and Mechatronic Engineering, The University of Sydney, Sydney, Australia. 